\title{
Prophylactic corneal cross-linking in LASIK surgery: effects on visual outcome and recovery time
}

\author{
Dominique Clare Oh${ }^{1}$, Yiong Huak Chan², Sao Bing Lee ${ }^{3}$, \\ Jovina Li Shuen See ${ }^{3}$ \\ ${ }^{1}$ Raffles Institution, Singapore, Singapore; ${ }^{2}$ Biostatistics Unit, Yong Loo Lin School \\ of Medicine, Singapore, Singapore; ${ }^{3}$ Shinagawa Eye Centre, Singapore, Singapore
}

\begin{abstract}
Introduction: Collagen cross-linking is a useful adjunct in preventing corneal ectasia after laser-assisted in situ keratomileusis (LASIK). This study aimed to evaluate whether prophylactic cross-linking in IntraLase LASIK affects optimum visual outcome and recovery time in the immediate post-surgery period and is associated with any side effects.
\end{abstract}

Methods: This was a retrospective case study on the right eyes of 100 Chinese subjects aged 18 to 40 years who underwent IntraLase LASIK. Fifty subjects who underwent cross-linking after completing LASIK (Group A) were compared with 50 subjects who did not undergo LASIK (Group B). Cases were evaluated for pre- and post-operative spherical equivalent, uncorrected distance visual acuity (UDVA) and corrected distance visual acuity (CDVA), recovery time and presence of side effects.

Results: At 1 week post-LASIK, mean (SD) UDVA of Group A subjects was poorer than Group $B$, at 1.05 (0.19) vs 1.17 (0.19) ( $p=0.036)$; however, there was no significant difference in CDVA ( $p=0.095)$. By 1 month post-LASIK, differences in both UDVA and CDVA were insignificant ( $p=0.055,0.106$, respectively). Mean recovery time was 2.72 (95\% confidence interval $[C l]=0.64-4.7)$ days longer in Group $A(p=0.010)$, although by 1 month post-LASIK, both groups were able to achieve CDVA equal to or better than that achieved pre-LASIK. Incidence of mild inflammation and dry eyes post-LASIK was similar in both groups ( $p=1.00,0.749$, respectively); no other complications were observed.

Conclusion: No differences in visual outcomes at and occurrence of side effects at 1 month post-LASIK were observed between subjects who underwent cross-linking prior to refractive surgery and those who did not. However, the group that underwent cross-linking had a slightly longer mean recovery time. Our study supports prophylactic cross-linking as a safe procedure that does not affect immediate visual outcomes among the Chinese population when used in adjunct with LASIK surgery.

Keywords: collagen cross-linking, corneal ectasia, corneal refractive surgery, LASIK

Correspondence: Dominique Clare Oh

E-mail: niqueclareoh@yahoo.com.sg 


\section{Introduction}

Corneal ectasia is a serious complication that can arise after corneal refractive surgery as a result of corneal weakening due to thinning of the corneal stroma. ${ }^{1}$ Patients who develop corneal ectasia may experience increased myopia and/ or astigmatism, associated with a loss of uncorrected and sometimes even best-corrected visual acuity. ${ }^{2,3}$ This is accompanied by characteristic keratometric steepening, in particular an asymmetric inferior corneal steepening, that is evident following corneal refractive surgery. ${ }^{3}$ Significant risk factors for corneal ectasia include the presence of forme fruste keratoconus pre-operatively, a high degree of myopia or astigmatism correction performed during corneal refractive surgery, and low residual stromal bed thickness of the cornea post-operatively. ${ }^{4}$ The incidence of corneal ectasia has been reported to range from $0.04 \%$ to $0.66 \%{ }^{1,4-6}$

Corneal collagen cross-linking has been performed for close to two decades since 1998 in patients with established corneal ectasia, such as in keratoconus patients. The technique originally involved the application of riboflavin and use of a photosensitizer, with exposure to ultraviolet (UV) light for 2 hours in order to form new chemical bonds between adjacent collagen fibrils in the cornea. ${ }^{7-10}$ Cross-linking in these patients has been widely reported to have a success rate of $70 \%$ in arresting corneal ectasia or, in some cases, even resulting in a $65 \%$ improvement of best-corrected visual acuity. ${ }^{10}$

In recent years, with the development of the Avedro cross-linking system, the necessary exposure time to UV light has been significantly shortened to just 1 to 10 minutes. This accelerated cross-linking procedure has been suggested to be safe and effective in halting post-operative laser-assisted in situ keratomileusis (LASIK) ectasia progression over a 1- to 2-year follow-up period, with patients achieving gains in refractive and keratometric stability, as well as uncorrected distance visual acuity (UDVA) and corrected distance visual acuity (CDVA). ${ }^{11-13}$ Thus, the use of corneal collagen cross-linking as a prophylactic corneal strengthening procedure in patients undergoing refractive surgery who are at higher risk of developing corneal ectasia has now been made possible.

Although the efficacy of the procedure may be inferred from aforementioned studies where cross-linking was performed in keratoconus patients, ${ }^{10}$ as well as in studies where less regression was observed in LASIK patients who underwent the procedure, ${ }^{11-13}$ it remains difficult to confirm actual efficacy in preventing corneal ectasia where cross-linking is performed as a prophylactic measure in patients undergoing elective corneal refractive surgery. In addition, it has been suggested that possible side effects of adjunctive corneal collagen cross-linking could include higher infection rates in the immediate post-operative period due to longer surgical exposure time and an increased risk of corneal scarring, 
infiltrates and diffuse lamellar keratitis, which may in turn affect the optimum visual outcomes of LASIK surgery or result in a longer recovery duration. Hence, we chose to evaluate the safety of the procedure in terms of immediate optimum visual outcomes and the time taken to achieve this outcome, as well as associated side effects during the immediate post-operative period. We believe looking at immediate optimum visual outcomes and length of recovery time are important not only in aiding patients to decide on whether to undergo this prophylactic adjunctive procedure but also to help refractive surgeons to provide better pre-operative counselling when advising patients on the procedure.

In our study, we looked at cross-linking using the Avedro system performed prophylactically in a Chinese population undergoing elective IntraLase LASIK surgery.

\section{Methods}

A total of 179 patients underwent IntraLase LASIK with cross-linking at the Shinagawa Eye Centre and a total of 601 patients underwent IntraLase LASIK without cross-linking between 1 July 2013 and 1 July 2014. From each of these two groups, an online random number generator was used to select 50 subjects. All subjects were Chinese, aged 18 to 40 years and treated by a single refractive surgeon. Subjects had undergone elective IntraLase LASIK using the iFS IntraLase femtosecond laser and the Schwind AMARIS 750 excimer laser machine. Corneal collagen cross-linking for the 50 subjects in Group A had been performed immediately after completion of IntraLase LASIK using VibeX Xtra (Riboflavin phosphate $2.80 \mathrm{mg} / \mathrm{ml}$ ). For IntraLase flap thickness of $120 \mu \mathrm{m}$, VibeX Xtra was applied to the corneal stroma for 65 seconds and after flap replacement, was activated by UV light for 66 seconds at $30 \mathrm{~mW} / \mathrm{cm}^{2}$, with a total energy of $2.0 \mathrm{~J} / \mathrm{cm}^{2}$.

In this retrospective study, the 50 subjects who had undergone Avedro cross-linking (Group A) were compared to the 50 subjects who had not undergone Avedro cross-linking (Group B). Case records were used to retrospectively evaluate the right eyes of all subjects in terms of visual outcomes, recovery duration and presence of side effects.

For all subjects, post-operative examinations were conducted at 1 day, 1 week and 1 month after LASIK surgery. The post-operative evaluation included UDVA and CDVA assessment using Snellen chart and clinical evaluation using slit-lamp examination for the presence of dry eye using tear breakup test and complications such as epithelial ingrowth and diffuse lamellar keratitis. Patients were also routinely asked whether they experienced any dry eye symptoms and were routinely treated with a tapering course of topical steroid (PredForte) and antibiotic (Cravit) eye drops in the first post-operative week. Subjects who were found to have diffuse lamellar keratitis on the first post-operative day were treated with 
additional topical Maxidex eye ointment at night in the first post-operative week.

Main outcomes evaluated were pre- and post-LASIK UDVA and CDVA, pre- and post-LASIK refraction and recovery time (defined as time taken to return to CDVA achieved pre-LASIK). A secondary outcome that was also evaluated was the presence of side effects such as post-operative diffuse lamellar keratitis, dry eyes, epithelial cell ingrowth, infection and other complications.

All analyses were performed using SPSS 24.0 with statistical significance set at $p<0.05$. Descriptive statistics for numerical variables were presented as mean (SD) and $n(\%)$ for categorical variables. Differences in post-operative numerical outcomes between the two groups were compared using two sample $T$-test when normality and homogeneity assumptions are satisfied, otherwise MannWhitney $U$ was performed; Fisher's exact test was used for categorical outcomes. Multivariate analyses using linear regression (for numerical outcomes) and logistic regression (for binary outcomes) adjusting for age, gender, pre-operative spherical equivalent and pre-operative CDVA as well as presence of dry eyes or inflammation were performed.

\section{Results}

Of the 50 Group A subjects who underwent cross-linking, 27 were female and 23 were male; the mean age (SD) was 28.3 (4.8) years. Pre-operatively, the mean spherical equivalent (SD) of Group A subjects was -6.2 (2.40) diopters (D) (range: -2.0 to -11.0$)$. Of the 50 Group $B$ subjects who did not undergo cross-linking, 25 were female and 25 were male; the mean age (SD) was 30.1 (5.8) years. Pre-operatively, the mean spherical equivalent (SD) was -4.68 (1.78) D (range: -1.5 to -9.3$)(p<0.001)$. There were no significant differences in age $(p=0.090)$ and gender ( $p=0.689)$ between the two groups.

At 1 week post-LASIK, visual outcome, measured by mean decimal UDVA, was not as good in Group A as compared to Group B. Mean decimal UDVA (SD) of Group A was 1.05 (0.19), while that of Group B was 1.17 (0.19) (adjusted $p=0.036$ ). The percentage achieving decimal UDVA of 1.0 or better was $84.0 \%$ and $94.0 \%$, respectively. However, while mean decimal CDVA (SD) was also slightly poorer in Group A at 1.10 (0.17) compared to $1.21(0.16)$ in Group B, the difference was not statistically significant (adjusted $p=0.095$ ). The percentage achieving decimal CDVA of 1.0 or better was similar at $96.0 \%$ and $100.0 \%$, respectively.

Furthermore, by 1 month post-LASIK, no significant difference in visual outcomes of LASIK surgery, in terms of both UDVA and CDVA, was observed. Mean decimal UDVA (SD) of Group A improved to 1.12 (0.18), while that of Group B improved to 1.21 (0.18) (adjusted $p=0.055$ ) (see Fig. 1). The percentage achieving decimal UDVA of 1.0 or better was the same in both groups at $94.0 \%$. Mean decimal CDVA of Group A and Group B was 1.17 (0.17) and 1.24 (0.15), respectively (adjusted 


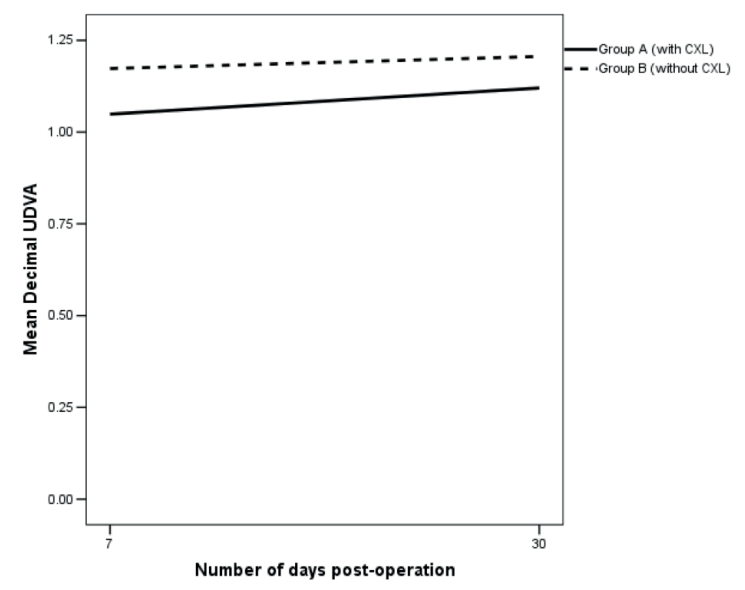

Fig. 1. Mean decimal UDVA from 1 week to 1 month post-LASIK.

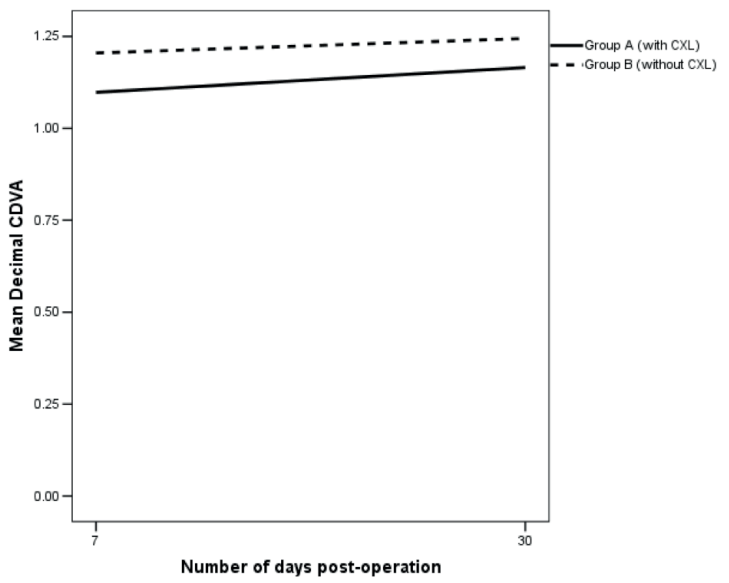

Fig. 2. Mean decimal CDVA from 1 week to 1 month post-LASIK.

$p=0.106$ ) (see Fig. 2), and $100.0 \%$ of subjects in both groups achieved decimal CDVA of 1.0 or better. Although mean UDVA and CDVA of Group A subjects remained slightly lower than Group B, differences were not statistically significant.

Mean recovery time (SD), defined as the time taken to return to CDVA achieved pre-LASIK, was longer for Group A subjects at 5.40 (5.9) days (range: 1-30 days) compared to 2.68 (2.7) days (range: 1-7 days) for Group B subjects, adjusted $p=0.010$. By 1 month post-LASIK, however, $100.0 \%$ of subjects in both groups 
were able to achieve decimal CDVA equal to or better than their pre-LASIK CDVA.

A similar percentage of subjects in both groups were found to have mild inflammation at 1 day post-LASIK ( $10 \%$ and $12 \%$, respectively, $p=0.749$ ) and $0.0 \%$ of subjects in both groups had inflammation at 1 week and 1 month post-LASIK. In addition, the percentage of subjects experiencing dry eyes post-LASIK was equal in both groups at $20.0 \%$. There were no other complications, such as diffuse lamellar keratitis, infection or epithelial cell ingrowth, in either group.

\section{Discussion}

A recent ex vivo study by Kanellopoullos et al. demonstrated that cross-linking combined with myopic LASIK provides significant increase in corneal stromal rigidity. ${ }^{13}$ In view of this biomechanical advantage, it is desirable to consider cross-linking as an adjunct to refractive surgery to reduce risk of developing corneal ectasia post-operatively. However, for cross-linking to be adopted as a prophylactic measure in refractive surgery, it is important to show that the procedure does not adversely affect visual outcomes and is not associated with any side effects.

Unlike previous studies that focused on evaluating the efficacy of prophylactic cross-linking in LASIK surgery for prevention of regression in visual outcomes due to corneal ectasia, our study looked at the effects of the procedure on the optimum visual outcome of LASIK surgery achieved within the immediate post-operative period, as well as the recovery time taken to achieve this outcome. Thus, instead of the longer 1- to 2-year follow-up period of other studies, we focused on the immediate 1 month post-surgery since $100.0 \%$ of subjects were able to achieve their pre-operative CDVA within this time period. Since cross-linking has already been suggested to be effective in slowing down regression, patients interested in the procedure would further benefit from knowing if it is associated with any adverse effects, such as infection or prolonged recovery time.

Our study found that prophylactic corneal collagen cross-linking in LASIK surgery may slightly increase the length of recovery time by an average 2.72 days and result in slightly poorer UDVA at 1 week post-LASIK. However, CDVA is not affected even at 1 week post-LASIK. Moreover, eventual optimum visual outcomes, in terms of both UDVA and CDVA, are unaffected by addition of the cross-linking procedure, as evident by how the optimum visual outcomes achieved in both groups at 1 month post-LASIK were similar. Combining the cross-linking procedure with LASIK surgery was also found to be safe as it did not increase the risk of any side effects such as diffuse lamellar keratitis, corneal scarring, dry eyes or epithelial cell ingrowth.

As this was a retrospective case study, however, one limitation was that subjects in the two groups differed in terms of their pre-operative refractive error, with 


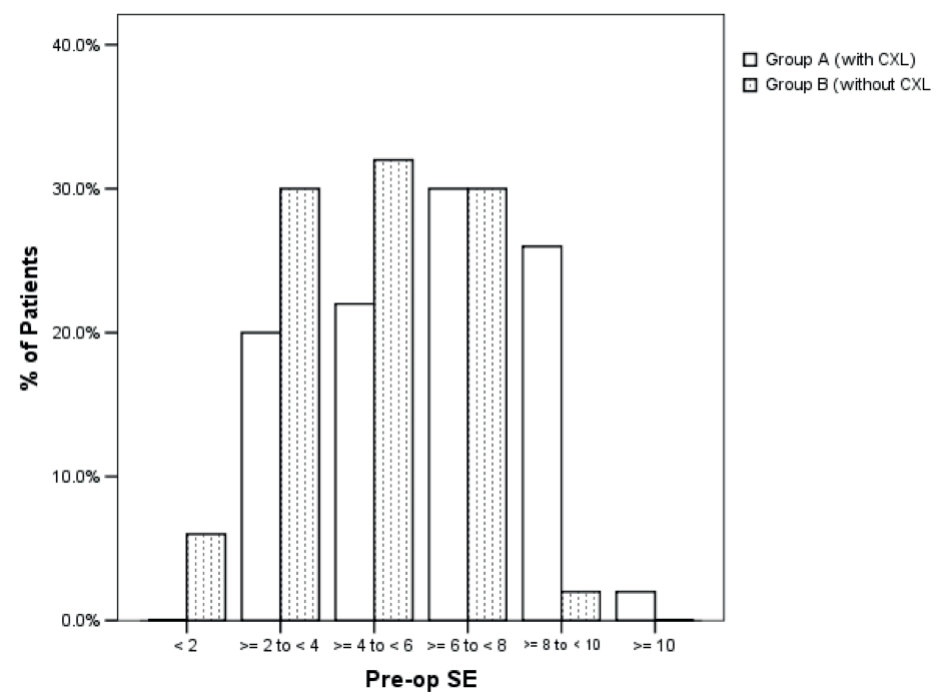

Fig. 3. Distribution of pre-operative spherical equivalent of Group A and Group B subjects.

Group A subjects having significantly higher mean refractive error $(p<0.001)$ (see Fig. 3). The proportion of high myopes was also higher in Group A; $58 \%$ had high myopia of $-6.00 D$ and above, compared to $32 \%$ of Group B $(p=0.009)$. Since refractive surgery for higher degrees of myopia necessitates a greater amount of ablation depth, which increases risk of corneal ectasia, more high myopes would have opted in for the cross-linking procedure, hence explaining why this group included more high myopes. Therefore, the slightly poorer visual outcome observed at 1 week post-LASIK in Group A may at least be partially attributed to the higher myopia present pre-operatively in these subjects, rather than only to the cross-linking procedure. As such, we tried to correct for these differences in our statistical analysis of results. Another limitation was that presence of side effects of dry eyes was clinically evaluated via the tear breakup test with only fluorescein dye and not using Schirmer's test. Lastly, although all patients managed to return to their pre-operative CDVA and make full recovery within the 1-month timeframe of our study, it could have been interesting to consider an additional follow-up at 3 months.

\section{Conclusion}

From our study, although mean recovery time was slightly longer in Group A subjects with cross-linking as compared to Group B subjects without cross-linking at 1 week post-LASIK, eventual visual outcomes at 1 month post-LASIK were 
similar. Furthermore, Group A subjects did not experience any additional complications, such as inflammation, dry eyes and epithelial cell ingrowth compared to Group B subjects. Therefore, our results support prophylactic cross-linking as a safe procedure to be used in adjunct with LASIK, with no adverse effects on immediate visual outcome, among the Chinese population.

To further investigate the effects of the cross-linking procedure, a prospective study involving a randomised controlled trial can be considered in future.

\section{References}

1. Rad AS, Jabbarvand M, Saifi N. Progressive keratectasia after laser in situ keratomileusis. J Refract Surg. 2004;20:S718-S722.

2. Twa MD, Nichols JJ, Joslin CE, et al. Characteristics of corneal ectasia after LASIK for myopia. Cornea. 2004 Jul;23(5):447-445. https://www.ncbi.nlm.nih.gov/pubmed/15220728.

3. Binder PS, Lindstrom RL, Stulting RD, et al. Keratoconus and corneal ectasia after LASIK [letter]. J Cataract Refract Surg. 2005;31:2035-2038.

4. Randleman JB, Russell B, Ward MA, Thompson KP, Stulting RD. Risk factors and prognosis for corneal ectasia after LASIK. Ophthalmology. 2003;110:267-275.

5. Pallikaris IG, Kymionis GD, Astyrakakis NI. Corneal ectasia induced by laser in situ keratomileusis. J Cataract Refract Surg. 2001;27:1796-1802.

6. Randleman JB, Woodward M, Lynn MJ, Stulting RD. Risk assessment for ectasia after corneal refractive surgery. Ophthalmology. 2008;115:37-50.

7. Spoerl E, Huhle M, Seiler T. Erhohung der Festigkeit der Horn haut durch Vernetzung. Ophthalmologe. 1997;94:902-906.

8. Spoerl E, Huhle M, Seiler T. Induction of cross-links in corneal tissue. Exp Eye Res. 1998;66:97-103.

9. Spoerl E, Schreiber J, Hellmund K. Untersuchungen zur Verfestigung der Hornhaut am kaninchen. Ophthalmologe. 2000;97:203-206.

10. Wollensak G, Spoerl E, Seiler T. Riboflavin/ultraviolet-A-induced collagenA cross-linking for the treatment of kertatoconus. Am J Ophthalmol. 2003;135:620-627.

11. Marino GK, Torricelli AA, Giacomin N, Santhiago MR, Espindola R, Netto MV. Accelerated corneal collagen cross-linking for postoperative LASIK ectasia: two-year outcomes. J Refract Surg. 2015 Jun; 31(6):380-384. http://www.ncbi.nlm.nih.gov/pubmed/26046704.

12. Kanellopoulos AJ, Asimellis G, Karabatsas C. Comparison of prophylactic higher fluence corneal cross-linking to control, in myopic LASIK, one year results. Clin Ophthalmol. 2014;8:2373-2381. https://www.dovepress.com/comparison-of-prophylactic-higher-fluence-corneal-cross-linkingto-con-peer-reviewed-fulltext-article-OPTH.

13. Kanellopoulos AJ, Asimellis G. Combined laser in situ keratometric mileusis and prophylactic high fluence corneal collagen cross-linking for high myopia: two-year safety and efficacy. J Cataract Refract Surg. 2015 Jul;41(7):1426-1433. http://www.jcrsjournal.org/article/ S0886-3350(15)00836-6/fulltext. 